\title{
Silencing of pantothenate kinase 2 reduces endothelial cell angiogenesis
}

\author{
FRANCESCA PAGANI $^{1}$, AKANSHA TRIVEDI ${ }^{1}$, DEEPAK KHATRI ${ }^{1}$, DANIELA ZIZIOLI ${ }^{1}$, \\ EMIRENA GARRAFA ${ }^{1,2}$, STEFANIA MITOLA $^{1}$ and DARIO FINAZZI ${ }^{1,2}$
}

\author{
${ }^{1}$ Section of Biotechnology, Department of Molecular and Translational Medicine, University of Brescia; \\ ${ }^{2}$ Clinical Chemistry Laboratory, Diagnostic Department, ASST-Spedali Civili di Brescia, I-25123 Brescia, Italy
}

Received May 9, 2018; Accepted August 23, 2018

DOI: $10.3892 / \mathrm{mmr} .2018 .9480$

\begin{abstract}
Coenzyme A (CoA) is an essential cofactor of cellular metabolism that is involved in $\sim 4 \%$ of cellular reactions. Its de novo production relies on five subsequent enzymatic steps, starting with the phosphorylation of vitamin B5. Pantothenate kinase 2 (PANK2) and coenzyme A synthase (COASY) catalyze the first and last steps of this pathway. Mutations in these genes lead to severe and progressive movement disorders, with neurodegeneration and iron accumulation in the basal ganglia, known as PANK2- and $C O A S Y$ protein-associated neurodegeneration, respectively. Given the ubiquitous role of CoA in cellular metabolism, it is still not clear why patients carrying PANK2 and COASY mutations develop almost exclusively neurological symptoms. Important clues are the energetic profile of neural cells as well as the high levels of PANK2 expression in the brain; however, other features may contribute to this selective tissue vulnerability. Notably, when pank2 or coasy expression was suppressed in zebrafish evident perturbation of neuronal development was observed, as well as severe defects in vasculature formation. Supplementation of CoA to fish water prevented the appearance of the phenotype, thereby confirming the specific connection with the availability of the metabolic cofactor. The present study investigated the associations between $P A N K 2$ defects and angiogenesis in a mammalian setting, and revealed that $P A N K 2$ expression was required for normal angiogenetic properties of human umbilical vein endothelial cells.
\end{abstract}

Correspondence to: Dr Daniela Zizioli, Section of Biotechnology, Department of Molecular and Translational Medicine, University of Brescia, Viale Europa 11, I-25123 Brescia, Italy

E-mail: daniela.zizioli@unibs.it

Key words: pantothenate kinase 2, pantothenate kinase 2-associated neurodegeneration, neurodegeneration with brain iron accumulation, coenzyme A, angiogenesis, human endothelial, umbilical vein cells

\section{Introduction}

In 2001, Zhou et al (1) associated the mutations in Pantothenate kinase 2 (PANK2) gene with a heterogenic neurologic disorder known as Hallervorden Spatz disease, later renamed as Pantothenate Kinase Associated Neurodegeneration (PKAN). It is a rare, autosomal recessive, neurodegenerative disease presenting iron deposition in the globus pallidus $(2,3)$ and included in the Neurodegeneration with Brain Iron Accumulation (NBIA) category (4). Based on age of onset and rate of progression, PKAN can be divided in a more common, early onset, classical form and a late onset, atypical one. While extrapyramidal signs such as dystonia, dysarthria and choreoatethosis, together with spasticity, cognitive decline and pigmentary retinopathy are common features of the classical form, parkinsonism and neuropsychiatric symptoms are usually observed in the atypical form. Magnetic resonance imaging is extremely useful at orienting the diagnosis since it usually reveals a sign known as 'eye of the tiger' that corresponds to iron deposition and tissue damage at the globus pallidus (5).

PanKs catalyze the phosphorylation of vitamin B5 in the first and essential step of coenzyme A (CoA) biosynthesis (6). Mammalians cells express up to four different forms of PanKs (7) and therefore it is not straightforward to understand the specific biochemical connections between defects in PANK2 functioning and the neurodegenerative process. Localization in mitochondria (8-11) together with specific biochemical properties may confer to the enzyme a pivotal role as a sensor for cellular CoA requirements $(12,13)$. Even though it is ubiquitously expressed, it is present at high relative levels in the brain $(1,12)$, thus suggesting a relevant role in neural cells. Studies performed in different experimental settings $(14,15)$ point to a reduction in cellular CoA level as the main consequence of PANK2 defects. This hypothesis is robustly supported by two facts: a) The rescue potential of molecules capable of bypassing the blockage in CoA biosynthesis, such as pantethine $(16,17), 4 \mathrm{P}$-pantetheine $(18,19)$ and CoA itself $(16,20)$; b) the recent identification of patients with clinical features largely overlapping to those of PKAN and carrying mutations in COASY gene, encoding the last enzyme of the same biochemical pathway $(21,22)$ and now linked to a new form of NBIA named COASY protein-associated Neurodegeneration (CoPAN). 
We recently developed zebrafish models of PKAN and CoPAN by microinjection of gene specific morpholinos (16). We described an essential role of pank2 in neuronal development, with pank 2 morphants showing severe perturbation of brain morphology and drastic reduction in the expression of fundamental transcription factors governing neuronal cell development and differentiation. Surprisingly, we also observed defects in the developing vasculature, with clear reduction in the intersegmental vessels (IVS) formation, structural alteration of the caudal plexus and caudal edema. We detected similar abnormalities also in coasy morphants, where we could also measure a reduction of CoA levels $(16,20)$. More importantly, all these features were efficiently rescued by providing panthetine (for pank2 morphants) or CoA (for both models) to fish water, thus confirming the specificity of the phenotype. The data let infer a selective sensibility of endothelial cells to pank 2 and CoA depletion. At the moment, there is no evidence indicating a role for vasculature defects in the pathogenesis of PKAN or CoPAN. These alterations were never investigated or documented in any other animal models. To evaluate the relevance of this data in mammalian settings, we analyzed the effects of PANK2 downregulation on the angiogenic properties of human umbilical vascular endothelial cells (HUVECs). Different experimental approaches clearly indicated that PANK2 is essential for HUVEC angiogenic activity, which in turn advocate a potential mechanism contributing to the development of PKAN pathology.

\section{Materials and methods}

Cells isolation, maintenance and transfection. HUVECs were previously isolated from umbilical veins according to an established protocol (23). Briefly, cord was washed with PBS containing penicillin $100 \mathrm{U} / \mathrm{ml}$ and streptomicin $100 \mu \mathrm{g} / \mathrm{ml}$. An injection needle was inserted into the umbilical vein and clamped; vein was washed twice with PBS using a 50cc syringe to remove residual blood clots and check for leaks. Then, a solution containing $0.1 \mathrm{U} / \mathrm{ml}$ of collagenase type I and $0.8 \mathrm{U} / \mathrm{ml}$ of dispase I (Sigma-Aldrich; Merck KGaA, Darmstadt, Germany) was injected in the vein and the cord was incubated at $37^{\circ} \mathrm{C}$. After 30', the liquid was collected and the vein was washed twice with PBS to collect all the detached cells. After centrifugation, the supernatant was removed; cells were suspended in growth medium and seeded on a collagen coated dish. Cells from 3 different cords were pooled, eventually frozen, and used at early passages (III-VII). HUVECs were cultured in EGM-2 ${ }^{\mathrm{TM}}$ Bullet Kit $^{\mathrm{TM}}$ (Lonza, Walkersville, MD, USA) supplemented with 10\% fetal bovine serum (Gibco; Thermo Fisher Scientific, Inc., Waltham, MA, USA), penicillin $100 \mathrm{U} / \mathrm{ml}$, streptomicin $100 \mu \mathrm{g} / \mathrm{ml}$ (BioSera, Nuaille, France) on collagen coated dishes. Cells were maintained at $37^{\circ} \mathrm{C}$ under $5 \% \mathrm{CO}_{2}$.

One set of three different 27-mer siRNAs duplexes specific for PANK2 gene was obtained from Origene (ID 80025; OriGene Technologies, Inc., Rockville, MD, USA). Out of them, the siRNA SR312773A was the most efficient and hence selected for all described experiments. We used a universal scrambled negative control siRNA duplex (SCR) from the same company as a negative control in all transfection experiments.

For transfection, $15 \times 10^{3} \mathrm{HUVECs} / \mathrm{cm}^{2}$ were seeded in 12-well or 6-wells plates. After $16 \mathrm{~h}$ they were incubated with specific and control siRNAs (1 $\mathrm{nM}$ final concentration) and 3 or $7.5 \mu \mathrm{l}$ of Lipofectamine ${ }^{\circledR}$ RNAiMax Transfection Reagent (Thermo Fisher Scientific, Inc.). After $5 \mathrm{~h}$ of incubation, the transfection medium was replaced by standard growth medium with or without $25 \mu \mathrm{M} \mathrm{CoA}$ (Sigma-Aldrich; Merck KGaA). Mock-treated cells received only the transfection reagent without the siRNA. After the transfection, cells were collected and analyzed or treated as described in the following section.

Biochemical and immunological methods. Equal amounts of protein extracts $(50 \mu \mathrm{g})$ obtained from HUVECs $48 \mathrm{~h}$ after the transfection were separated on SDS-PAGE and transferred to PVDF membrane. The membranes were incubated at $4^{\circ} \mathrm{C}$ for $16 \mathrm{~h}$ with a monoclonal mouse anti-PANK2 (1:500, no. TA501321; OriGene Technologies, Inc.) and a monoclonal mouse anti-actin $(1: 1,000$, no. TA811000; OriGene Technologies, Inc.). Membranes were washed three times with TBS $0,05 \%$ Tween and incubated for $1 \mathrm{~h}$ with HRP-conjugated anti-mouse IgG (1:5,000; Pierce; Thermo Fisher Scientific, Inc.). Immunocomplexes were detected by a chemiluminescence detection kit (Protein Detection System; Genespin, Milan, Italy) with Odissey ${ }^{\circledR}$ Imaging System (LiCor Bioscience, Lincoln, NE, USA). The densitometric analysis was performed with the Kodak 1D 3.6 program.

Cell viability/proliferation. At $24 \mathrm{~h}$ after the transfection, $15 \times 10^{3} \mathrm{HUVECs} / \mathrm{cm}^{2}$ were seeded in 12-well plates in triplicates. After $48 \mathrm{~h}$ the medium was collected and analyzed for the presence of dead cells. Adherent cells were detached with trypsin $0.1 \% /$ EDTA, washed and stained with trypan blue to check for vitality.

MTT assay. $48 \mathrm{~h}$ after the transfection, 5,000/well HUVECs were seeded in 96-well plates in triplicates. The day after, cells were washed and incubated with $0.5 \mathrm{mg} / \mathrm{ml}$ methyl-thiazol-tetrazolium (Sigma-Aldrich; Merck KGaA) in cell medium for $3.5 \mathrm{~h}$. The supernatant was then removed, the insoluble formazan blue dissolved in $75 \mu \mathrm{l}$ DMSO, and absorbance was measure at $540 \mathrm{~nm}$ with the EnSight Multimode Plate Reader (PerkinElmer, Inc., Waltham, MA, USA).

ATP measurement. ATP concentration was measured using the ATP luminescence assay kit (CellTiter-Glo Luminescent Cell Viability Assay; Promega Corporation, Madison, WI). $24 \mathrm{~h}$ after the transfection, 25,000 cells/well were transferred in a 96-well plate in triplicate and equilibrated at room temperature for $30 \mathrm{~min} .100 \mu \mathrm{l}$ of reagent were added in each well of the plate and the luminescent signal measured by the EnSight Multimode Plate Reader.

$R N A$ extraction and reverse transcription-quantitative polymerase chain reaction (RT- $q P C R)$. HUVECs were collected $48 \mathrm{~h}$ after the transfection and RNA was purified by using TriReagent solution (Sigma-Aldrich; Merck KGaA). $1 \mu \mathrm{g}$ of total RNA was used to synthesize the first strand of cDNA with the Improm-II Reverse Transcriptase (Promega Corporation), using oligo-dT $4 \mu \mathrm{M}$ as primer in a total volume of $20 \mu \mathrm{l} .1 \mu \mathrm{l}$ of the reaction was used as template for the real-time amplification. We set up triplicate reactions in a total volume of $10 \mu \mathrm{l}$, with GoTaq ${ }^{\circledR}$ qPCR Master Mix (Promega Corporation) and 
Table I. Sequences of primers used for reverse transcription-quantitative polymerase chain reaction analysis.

\begin{tabular}{lll}
\hline Gene & \multicolumn{1}{c}{ Forward primer $\left(5^{\prime}-3^{\prime}\right)$} & \multicolumn{1}{c}{ Reverse primer $\left(5^{\prime}-3^{\prime}\right)$} \\
\hline HPRT1 & TGTTTTCCTTGGTCAGGCAG & AAGCTTGCGACCTTGACCAT \\
PANK1 & AGGTGTCAGCATTCTAGCCG & GGTCTCACAACCAGTCAGCA \\
PANK3 & GCACACAAGCTGACAAGCTG & ACATTCGTGCCACAGAACCA \\
VE-CADHERIN & GCTCCCCTCCAAAGACGGTCG & AACAGATCGTGCCTGTGGGCTGAGG \\
VEGFR2 & GAACATTTGGGAAATCTCTTGC & CGGAAGAACAATGTAGTCTTTGC \\
CD31 & TCCACATCAGCCCCACCGGA & TGGGCCACAATCGCCTTGTCC \\
FGFR1 & GGGCTGGAATACTGCTACAA & GCCAAAGTCTGCTATCTTCATC
\end{tabular}

HPRT1, hypoxanthine guanine phosphoribosyl transferase 1; PANK, pantothenate kinase; VE-CADHERIN, vascular endothelial-cadherin; VEGFR2, vascular endothelial growth factor receptor 2; CD31, cluster of differentiation; FGFR1, fibroblast growth factor receptor 1.

$0,5 \mu \mathrm{M}$ of the specific primers. The reaction was run on Eco 48 PCR Real-time Machine (PCR max, Staffordshire, ST15 0SA, $\mathrm{UK}$ ) for 40 cycles of $95^{\circ} \mathrm{C}$ for $10 \mathrm{sec}$ and $60^{\circ} \mathrm{C}$ for $20 \mathrm{sec}$. Hypoxanthine guanine phosphoribosyl transferase 1 (HPRT1) mRNA was used as endogenous reference for the relative quantification, which was performed by the $\Delta \Delta \mathrm{Cq}$ method, as previously described $(24,25)$. For human PANK2 mRNA amplification we used a PrimeTime ${ }^{\circledR}$ qPCR Assay primers mix located on exon 5-6 (IDT Technologies Inc., Coralville, IA, USA). The primers for the other genes are shown in Table I. For the comparison of the amount of different PanK mRNA isoforms in HUVECs, the ratio between the quantification cycle $(\mathrm{Cq})$ of each PanK gene and the Cq of HPRT1 gene was calculated. Since the $\mathrm{Cq}$ inversely correlates with the amount of target mRNA and the HPRT1 Cq value is the same for all samples, the highest ratio value corresponds to the lowest level of target mRNA.

Cell motility assay. Cell motility was assessed by time-lapse video-microscopy. Briefly, 5,000 transfected cells were seeded in 12-well plate and stimulated with $30 \mathrm{ng} / \mathrm{ml} \mathrm{VEGF} \mathrm{or} 2$ and $10 \% \mathrm{FBS}$. Constant temperature $\left(37^{\circ} \mathrm{C}\right)$ and $5 \% \mathrm{CO}_{2}$ were maintained throughout the experimental period by means of a heatable stage and climate chamber. Cells were observed under an inverted photomicroscope (Zeiss Axiovert $200 \mathrm{M}$ ) and phase-contrast snap photographs (one every $10 \mathrm{~min}$ ) were digitally recorded for $5 \mathrm{~h}$. Cell paths (20-30 cells per experimental point) were generated from centroid positions and migration parameters were analysed with the 'Chemotaxis and Migration Tool' of ImageJ Software (rsbweb.nih.gov/ij).

Wound healing scratch assay. HUVECs were transfected as described earlier. After $48 \mathrm{~h}$ (when confluency was reached), wounds were created in the cellular monolayers with a $1.0-\mathrm{mm}$ wide rubber policeman. An image was captured and the surface area of the wound was delimited by a rectangular shape with ImageJ Software. Then, cells were incubated in fresh medium with 5\% FCS. After $10 \mathrm{~h}$, cell monolayers were fixed with $2.5 \%$ glutaradehyde, stained with crystal violet $0.1 \%$ and photographed. Endothelial cells invading the rectangular area (same size in all samples) delimiting the original wound were counted by computer-assisted analysis of the digitalized images with ImageJ Software (rsbweb.nih.gov/ij).
Morphogenesis on three-dimensional gels. $10 \mu \mathrm{l} /$ well of Geltrex $^{\mathrm{TM}}$ (Thermo Fisher Scientific Inc.) was used to coat 15-well $\mu$-Slide Angiogenesis plates (IBIDI, Martinsried, Germany) at $4^{\circ} \mathrm{C}$. After gelification at $37^{\circ} \mathrm{C}, 10,000$ transfected HUVECs were seeded at least in triplicate onto coated dishes in 5\% FBS cell growth medium. Newly formed endothelial cell 'cords' and 'tubes' were photographed after $20 \mathrm{~h}$ at $40 \mathrm{X}$ magnification (Olympus IX51 inverted microscope) and empty areas were counted by two investigators without knowledge of the samples tested.

Statistical analyses. One-way analysis of variance with Dunnett's post hoc test were applied to compare the different groups. The results were analyzed by GraphPad Prism 6.01 software (GraphPad Software, Inc., La Jolla, CA, USA) and $\mathrm{P}<0.05$ was considered to indicate a statistically significant difference. Data were presented as the mean \pm standard deviation of at least 3 independent experiments, unless otherwise stated in the figure legend.

\section{Results}

We first analyzed the expression of different $P A N K$ isoforms in HUVECs by RT-qPCR. PANK2 mRNA level was the highest, together with $P A N K 3$ mRNA, while $P A N K 1$ was expressed at lower level (Fig. 1A). This condition is also observed in human brain (12). To characterize the role of PANK2 in HUVECs we downregulated its expression by siRNA technology. We selected the best performing siRNA out of three different molecules and identified the concentration of $1 \mathrm{nM}$ as the one providing the best ratio between silencing efficiency and absence of off target effects (data not shown). At this low dose of specific siRNA, we obtained a significant downregulation of PANK2 mRNA levels (approximately 75\%) as measured by RT-qPCR $48 \mathrm{~h}$ after the transfection (Fig. 1B). We did not observe any change in PANK1 and PANK3 mRNAs amount in these cells and transfection with an unrelated, control siRNA (SCR) resulted in no modification of the expression of any of the PANK genes (Fig. 1B). The immunoblotting performed with protein extracts collected $48 \mathrm{~h}$ after the transfection confirmed the decrease in the synthesis of PANK2 protein, with a reduction of the bands corresponding to precursor $(63 \mathrm{kDa})$, 

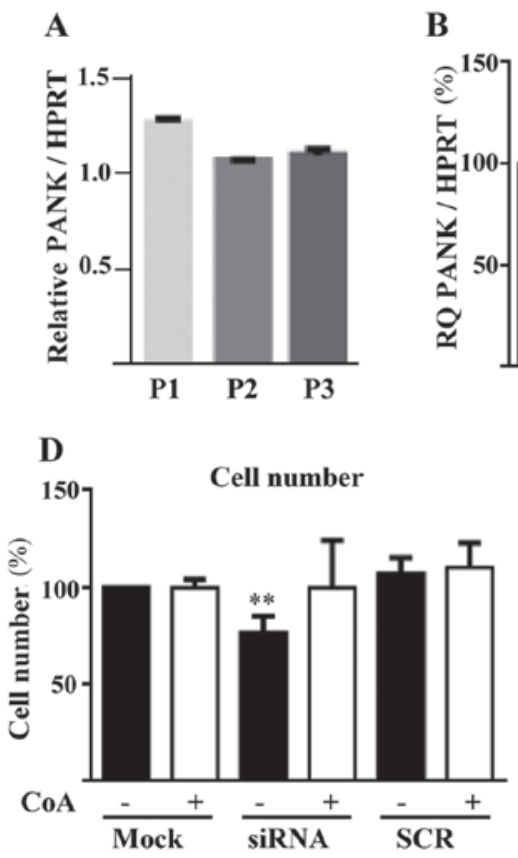

B

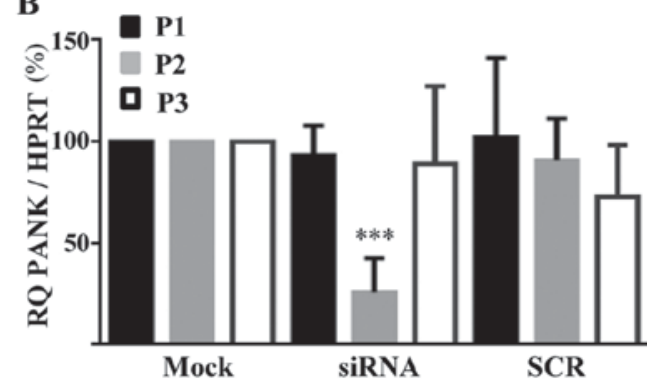

$\mathbf{E}$

$\mathbf{F}$

C
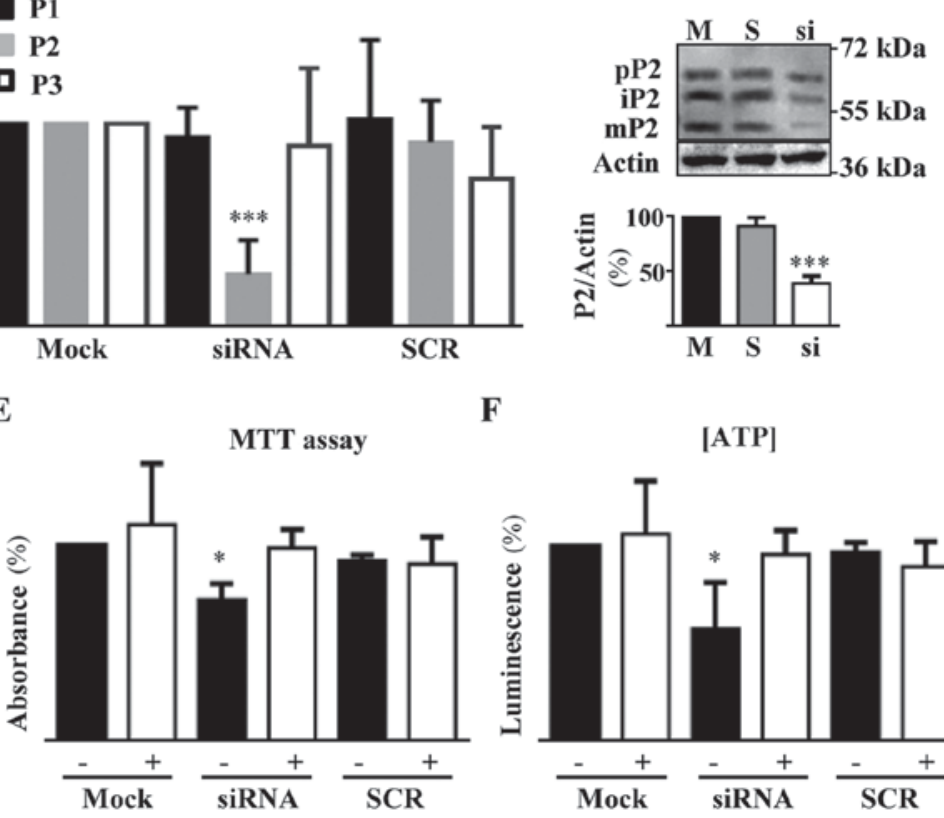

[ATP]

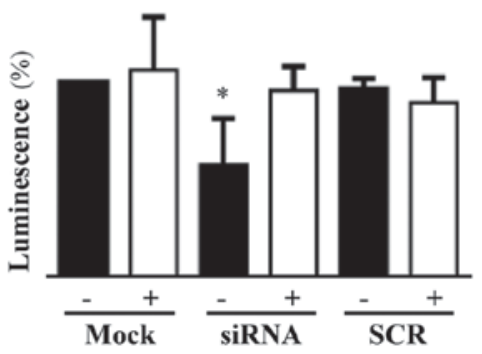

Figure 1. PANK2 expression and silencing in HUVECs. (A) RT-qPCR quantification of the expression of different PanKs isoforms in HUVECs. The Y-axis presents the ratio between the relative expression of each PanK and HPRT1 gene. (B) RT-qPCR evaluation of efficiency and specificity of PANK2 siRNA silencing. (C) Representative immunoblotting ( $\mathrm{n}=3$ ) and quantification of human PANK2 protein in control and silenced HUVECs. (D) Quantification of cell number, (E) viability (MTT Assay), and (F) ATP content in HUVECs transfected with PANK2 and control (SCR group) siRNA. "P $<0.05,{ }^{* * *} \mathrm{P}<0.01$ and ${ }^{* * * *} \mathrm{P}<0.001$ vs. Mock. P1, $\mathrm{P} 2$, and $\mathrm{P} 3$ indicate PANK1, PANK2, and PANK3, respectively; $\mathrm{pP} 2$, iP2, and mP2 indicate precursor, immature, and mature PANK2, respectively. SCR, scrambled negative control siRNA duplex; RT-qPCR, reverse transcription-quantitative polymerase chain reaction; PANK2, pantothenate kinase 2; HUVECs, human umbilical vascular endothelial cells; ATP, adenosine triphosphate; HPRT1, hypoxanthine guanine phosphoribosyl transferase 1; siRNA, small interfering RNA; CoA, Coenzyme A.

immature $(59 \mathrm{kDa})$ and mature forms $(48 \mathrm{kDa})$ of the protein. Altogether, PANK2 protein level was decreased by more than $60 \%$ in the silenced cells as compared to mock- and SCR-transfected cells (Fig. 1C). To verify the biochemical consequences of PANK2 silencing, we analyzed parameters known to be perturbed by downregulation of the gene in other cell types $(26,27)$, such as cell proliferation and ATP content. The same number of cells was plated, exposed to the silencing reaction and counted after $72 \mathrm{~h}$. Silenced cells were approximately $25 \%$ less than mock- and SCR-transfected ones $(\mathrm{P}<0.01$; Fig. 1D). Since we did not find signs of cell death in any sample, the difference should represent a reduction of the proliferation rate. To assess vitality and energetic profile of silenced cells we performed the MTT assay and measured ATP content. PANK2-silenced cell showed significant reduction of the dehydrogenase activity and of ATP content $(\mathrm{P}<0.05$; Fig. $1 \mathrm{E}$ and $\mathrm{F})$. All the features associated with $P A N K 2$ downregulation were prevented by the addition of $25 \mu \mathrm{M} \mathrm{CoA}$ to the cell medium $5 \mathrm{~h}$ after the transfection. This indirectly suggests that PANK2 silencing leads to depletion of cellular CoA levels and confirms the specificity of our experimental setting. Altogether this set of analyses shows that the reduction of PANK2 expression in HUVECs induces well known features of energetic and mitochondrial defects and reduced proliferation.

Then, we moved to the analysis of the angiogenic properties of control and silenced HUVECs. Firstly, we evaluated single cell motility by time-lapse video-microscopy. $24 \mathrm{~h}$ after the transfection, 5,000 cells/well were seeded in triplicate in a 12-well plate, stimulated with either $30 \mathrm{ng} / \mathrm{ml}$ VEGF, or

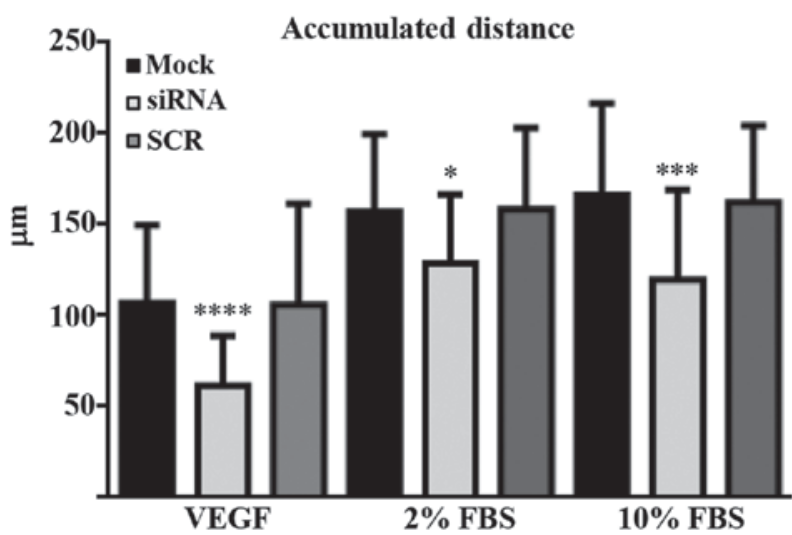

Figure 2. Silencing of $P A N K 2$ reduces single cell motility. A total $24 \mathrm{~h}$ post-treatment with $P A N K 2$-specific and control small interfering RNA (scrambled negative control small interfering RNA duplex), human umbilical vascular endothelial cells were seeded in 12-well plates at least in 3 times, stimulated with VEGF $30 \mathrm{ng} / \mathrm{ml}, 2$ or $10 \%$ FBS and recorded for $5 \mathrm{~h}$. The migration of $>30$ cells/well was analyzed using the 'Chemotaxis and Migration Tool' of ImageJ Software and expressed as the accumulated distance. Data are presented as the mean \pm standard deviation. ${ }^{*} \mathrm{P}<0.05$, ${ }^{* * * *} \mathrm{P}<0.001$ and ${ }^{* * * *} \mathrm{P}<0.0001$ vs. Mock. PANK2, pantothenate kinase 2; VEGF, vascular endothelial growth factor; FBS, fetal bovine serum.

10 or $2 \%$ FBS and observed under an inverted photomicroscope. Phase-contrast photographs were taken every $10 \mathrm{~min}$ for $5 \mathrm{~h}$ and the migration pattern of at least 30 cells/well was determined by the 'Chemotaxis and Migration Tool' of ImageJ Software. As shown in Fig. 2A, the accumulated distance of PANK2-silenced HUVECs treated with VEGF 
A
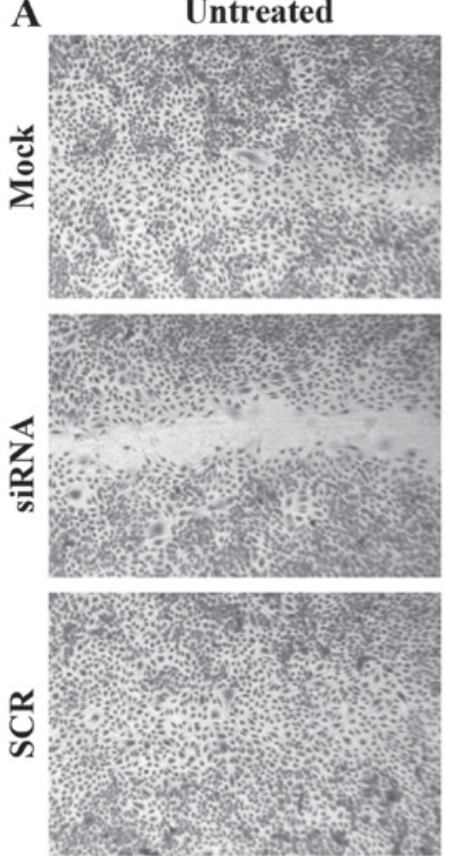

B

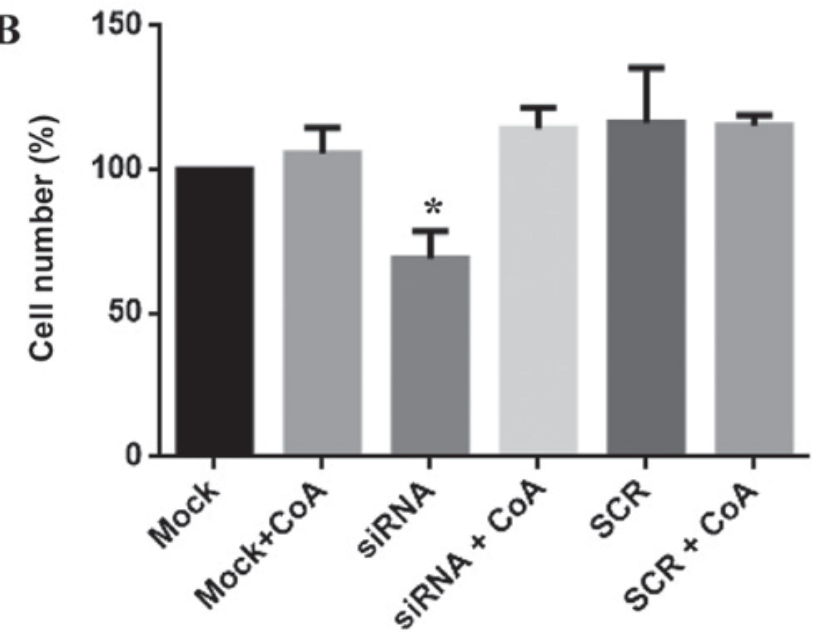

Figure 3. Silencing of $P A N K 2$ reduces the wound healing capability of human umbilical vascular endothelial cells. Wound healing scratch assays were performed with silenced cells, in the absence or presence of CoA $30 \mu \mathrm{M}$. (A) Micrographs of the extent of wound healing obtained following $10 \mathrm{~h}$ in mock, PANK2- and SCR-siRNA samples. Three independent experiments were performed and representative results are presented (magnification, x 50). (B) The migration efficiency of cells in different samples was assessed by counting the number of cells in the rectangular area delimiting the surface of the original wound. Data are presented as the mean \pm standard deviation. ${ }^{*} \mathrm{P}<0.05$ vs. Mock. PANK2, pantothenate kinase 2; CoA, coenzyme A; siRNA, small interfering RNA; SCR, scrambled negative control siRNA duplex.

was greatly reduced $(58 \%)$ as compared to those of mockand SCR-transfected cells $(\mathrm{P}<0.001)$. Similar results were obtained when cells were treated with 10 or $2 \%$ FBS instead of VEGF (Fig. 2). Subsequently, we analyzed the collective migration performance of PANK2-deficient cells by the wound healing scratch assay. As shown in Fig. 3A, the wound was hardly visible in the wells with mock- and SCR-treated cells after $10 \mathrm{~h}$ of incubation, whereas it was still evident in the wells of PANK2-silenced HUVECs. We counted the cells migrated towards the wound and found that much fewer cells (reduction of approximately 30\%) invaded the region of the
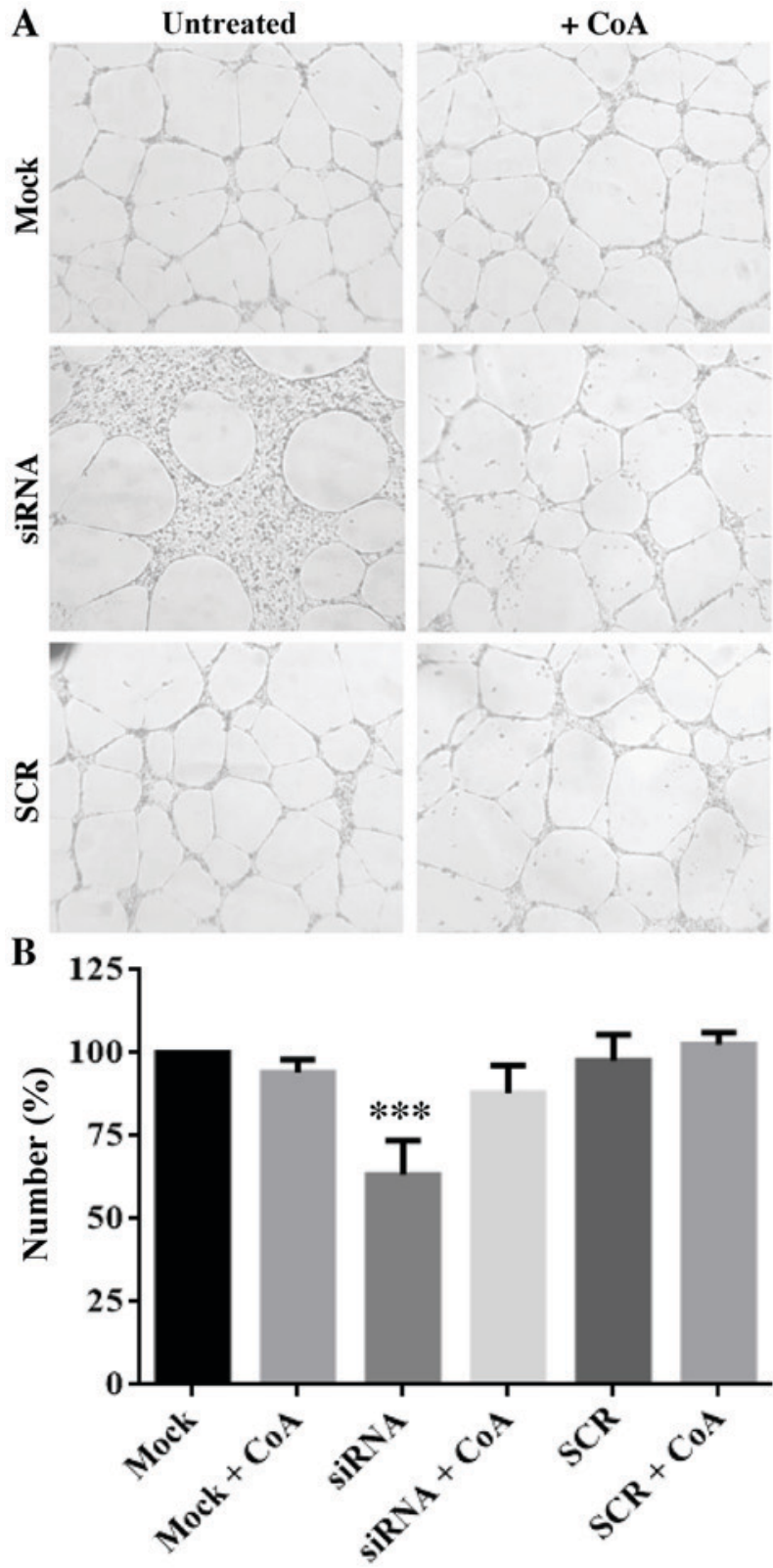

Figure 4. Silencing of PANK2 affects the tube formation ability of HUVECs. Silenced HUVECs treated with or without with CoA $30 \mu \mathrm{M}$ were seeded at a low density onto coated dishes in 5\% fetal bovine serum growth medium and photographed following $20 \mathrm{~h}$. (A) Representative micrographs (magnification, x40). (B) Quantification of empty areas in different samples. Data are presented as the mean \pm standard deviation of three independent experiments. ${ }^{* * * *} \mathrm{P}<0.001$ vs. Mock. HUVECs, human umbilical vascular endothelial cells; PANK2, pantothenate kinase 2; CoA, coenzyme A; siRNA, small interfering RNA; SCR, scrambled negative control siRNA duplex.

wound at the end of the incubation when silenced for PANK2 in comparison to mock- and SCR-treated cells (Fig. 3B). The treatment with $25 \mu \mathrm{M}$ CoA restored the normal migration also in this case. Finally, we evaluated the capacity of the cells to form capillary-like structures in a three-dimensional gel (tube formation assay). We seeded 10,000 cells in triplicate in wells coated with Matrigel and counted the number of areas fully delimited by cell cords/tubes (empty areas) after $20 \mathrm{~h}$ of incubation at $37^{\circ} \mathrm{C}$. Once again, we observed a net reduction (approximately $40 \%, \mathrm{P}<0.001$ ) in the number of empty areas delimited by $P A N K 2$-silenced cells, suggesting 


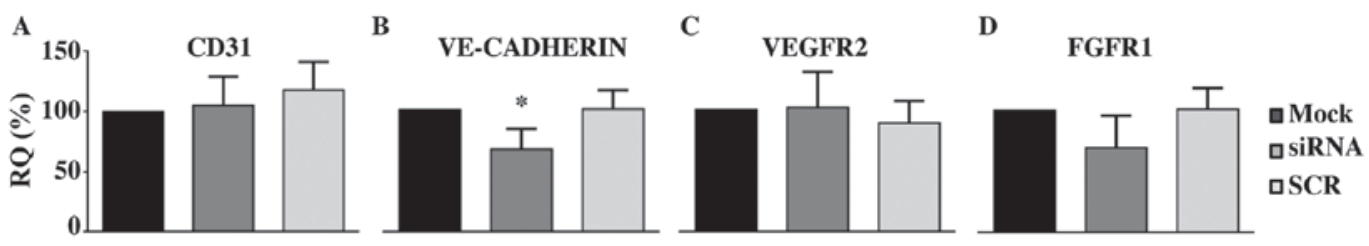

Figure 5. Silencing of PANK2 decreases VE-CADHERIN expression in human umbilical vascular endothelial cells. Silenced cells were collected $48 \mathrm{~h}$ post-transfection, RNA was extracted and the expression of endothelial markers and proliferative factors were analyzed by reverse transcription-quantitative polymerase chain reaction. Hypoxanthine guanine phosphoribosyl transferase 1 was used the reference gene. The relative expression of each target mRNA was calculated as a percentage of that obtained in the mock-treated cells. (A) CD31, (B) VE-CADHERIN, (C) VEGFR2 and (D) FGFR1. Data are presented as the mean \pm standard deviation of three independent experiments. "P<0.05 vs. Mock. PANK2, pantothenate kinase 2; siRNA, small interfering RNA; SCR, scrambled negative control siRNA duplex; CD31, cluster of differentiation; VE-CADHERIN, vascular endothelial cadherin; VEGFR2, vascular endothelial growth factor receptor 2; FGFR1, fibroblast growth factor receptor 1; RQ, relative quantification.

that downregulation of $P A N K 2$ inhibits not only the motility of HUVECs, but also their ability to reorganize in tube-like structures. Again, the addition of CoA to the medium restored the values observed in control and SCR-treated cells (Fig. 4). To investigate possible molecular mechanism linking the silencing of PANK2 with the defects of the angiogenic properties of HUVECs, we analyzed the expression level of different genes known to play a major role in the proliferative/migration response of endothelial cells by using RT-qPCR. We did not detect any difference in the level of CD31, a typical marker of endothelial cells (Fig. 5A), thus indicating the conservation of main endothelial features in transfected cells. The relative mRNA amount of two major receptors involved in the response to proliferative stimuli, Vascular Endothelial Growth Factor Receptor 2 (VEGFR2) and Fibroblast Growth Factor receptor 1 (FGFR1) was also unchanged (Fig. 5C and D). Interestingly, we detected a moderate decrease of VE-CADHERIN expression level (Fig. 5B), which was approximately $60 \%$ of that of mock- and SCR-treated cells $(\mathrm{P}<0.05)$.

\section{Discussion}

Mutations in PANK2 lead to the development of PKAN, a movement disorder included in the NBIA category. It is highly probable that a shortage of CoA represents the initial pathogenic trigger, even though direct measurements in patients are still missing. CoA and its acyl-derivatives are fundamental cofactors for a large number of biochemical reactions in all types of cells and tissues; it is remarkable and poorly understood that the clinical features of PKAN are essentially limited to the central nervous system. When we observed severe perturbations of vasculature development in zebrafish embryos with downregulation of pank2 expression, we reasoned that it could be a feature limited to this experimental model. Zebrafish embryos develop externally and essentially rely on the inherited pool of vitamin B5 and CoA for many days of growth. Minor perturbations in this delicate balance may induce more severe consequences in Danio rerio embryos than in other systems, where external supply of these metabolites may support compensatory mechanisms. At the same time, assuming that human and zebrafish pantothenate kinases have similar biochemical properties, the relevance of pank2 in CoA biosynthesis could be increased in zebrafish embryos due to a limited reservoir of acetyl-CoA, a potent inhibitor of PANK2 activity (13).
Nonetheless, we investigated the effects of PANK2 silencing in mammalian cells and found that the reduction of PANK2 protein severely affects the angiogenetic properties of HUVECs. Different types of analysis evidenced defects in migration and cord formation capability in PANK2-silenced cells as compared to control. This was associated with features, such as lower proliferation rate and energetic defects, which are commonly observed in other cells upon PANK2 suppression $(16,26,28)$. The addition of CoA to the medium immediately after the transfection prevented all these alterations efficiently, indirectly indicating a selective decrease in CoA availability. It could well be that the defect in HUVECs angiogenic properties is a direct consequence of the mitochondrial dysfunction (MTT assay) and the lower amount of ATP, but recent studies pinpoint a more direct role of acetyl-CoA and fatty acid oxidation (FAO) in controlling angiogenesis and cell differentiation, even in the absence of significant energetic defects. HUVECs cultured in charcoal-stripped serum (CSS) showed impaired proliferation and angiogenic properties, which were largely restored by treatment with palmitic acid or acetyl-CoA precursor acetate (29). The same molecules reestablished normal cell proliferation and sprouting activity in ECs (30) and lymphangiogenesis in lymphatic ECs (31), suffering from pharmacological or genetic blockage of FAO. Thus, it seems plausible that acetyl-CoA (and indirectly CoA), exerts a control on cell fate and activity through the direct connection with fatty acid metabolism and independent of energetic changes. Since PANK2 represents an important link between acetyl-CoA levels and FAO (13), its loss of function may have direct consequences on cellular programs controlling proliferation and differentiation. Mechanisms such as altered nucleotide production (30) and modification of histone acetylation (31) may contribute to this phenomenon. We documented a reduction of VE-CADHERIN mRNA levels in silenced HUVECs. We observed a similar reduction, together with that of flil, in pank 2 and coasy morphants $(17,20)$. It is known that the decrease of cellular CoA availability affects histones 3 and 4 acetylation (27); this could result in modification of transcriptional processes and lower mRNA level of VE-CADHERIN. A thorough analysis of the transcriptional changes associated with PANK2 defects could shed more light onto downstream, synergistic processes participating in disease development.

Alterations in angiogenesis may concur to neurodegenerative processes (32) and represent a potential target for 
therapeutic strategies (33). Our data seem to support such an involvement in PKAN or CoPAN. The replication in more appropriate experimental models for brain microvasculature endothelial cells as well as in other systems such as Pank2 $2^{-1-}$ mice and endothelial cells derived from patients induced Pluripotent Stem cells could be ways to further investigate this hypothesis. Albeit limited by the extreme paucity of cases, the histological analysis of brain samples from patients could provide more definitive arguments. Noteworthy, the globus pallidus of PKAN patients presents numerous proteinaceous aggregates enriched with ubiquitin and apolipoprotein $\mathrm{E}$ and most probably representing remnants of degenerated neurons (34). Similar lesions were detected in non-PKAN brains at and around areas of infarct involving large GABAergic neurons, such as Purkinje cells or those found in the globus pallidus and the substantia nigra, pars reticulata. These aggregates represent ischemic injuries and may suggest the involvement of hypoxia in PKAN pathogenesis. Metabolic changes in both neuronal and endothelial cells with altered CoA biosynthesis and eventually microvasculature defects may concur to the establishment of a hypoxic condition that leads to neuronal cell death and neurodegeneration. Both cellular (large GABAergic neurons with long axonal extension) and tissue [poor vascularization (35), high iron content] features may contribute to the specific susceptibility of the globus pallidus to reduced CoA availability due to PANK2 mutations.

Beside this initial indication regarding the implication for vasculature development and maintenance, our analysis strengthens once again the essential role of PANK2 in cellular CoA homeostasis. Like neurons, HUVECs express different isoforms of PANK, with PANK2 and PANK3 being the most abundant at the mRNA level. With the intent to limit any possible off-target effects, we applied low doses of siRNA and obtained approximately 75 and $60 \%$ reduction in mRNA and protein level, respectively. This is clearly a limit of our approach: the residual enzymatic activity could mitigate the severity of the phenotype and facilitate the rescue efficiency of CoA. Nonetheless, the phenotypic changes we observed were highly reproducible, suggesting that neither PANK3 nor PANK1 can compensate for PANK2 defects in HUVECs. Even though partial explanation comes from the specific localization of PANK2 in the mitochondria and the differences in the regulatory properties of the enzyme (13), this aspect remains poorly understood and its comprehension could provide more insight into the specific neuronal vulnerability and the mechanisms underpinning the neurodegenerative process. To this aim, we are editing the genome of mammalian cells and fish to generate models with PANK2 null alleles. They will be important tools to investigate cellular mechanisms of CoA deficiency and possible correction strategies.

\section{Acknowledgements}

The authors are grateful to Dr Elisabetta Crescini (University of Brescia, Brescia, Italy) for their technical assistance with the isolation and culture of HUVECs, and to Professor Patrizia Dell'Era (University of Brescia) for assisting with some of the experiments.

\section{Funding}

The present study was supported by Health and Wealth (ZEBRACA) and ex60\% funds from University of Brescia.

\section{Availability of data and materials}

The datasets used and/or analyzed during the current study are available from the corresponding authors on reasonable request.

\section{Authors' contributions}

FP designed and performed the experiments, analyzed the data, and wrote and revised the manuscript. AT, DK, EG and SM assisted with the study design, performed some of the experiments and revised the manuscript. DZ and DF conceived and designed the experiments, analyzed the data, prepared the figures, and wrote and revised the manuscript.

\section{Ethics approval and consent to participate}

Not applicable.

\section{Patient consent for publication}

Not applicable.

\section{Competing interests}

The authors declare that they have no competing interests.

\section{References}

1. Zhou B, Westaway SK, Levinson B, Johnson MA, Gitschier J and Hayflick SJ: A novel pantothenate kinase gene (pank2) is defective in hallervorden-spatz syndrome. Nat Genet 28: 345-349, 2001.

2. Angelini L, Nardocci N, Rumi V, Zorzi C, Strada L and Savoiardo M: Nhallervorden-spatz disease: Clinical and mri study of 11 cases diagnosed in life. J Neurol 239: 417-425, 1992.

3. Swaiman KF: Hallervorden-Spatz syndrome and brain iron metabolism. Arch Neurol 48: 1285-1293, 1991.

4. Hayflick SJ, Westaway SK, Levinson B, Zhou B, Johnson MA, Ching KH and Gitschier J: Genetic, clinical, and radiographic delineation of hallervorden-Spatz syndrome. N Engl J Med 348: 33-40, 2003.

5. Hayflick SJ, Hartman M, Coryell J, Gitschier J and Rowley H: Brain MRI in neurodegeneration with brain iron accumulation with and without PANK2 mutations. Ajnr Am J Neuroradiol 27: 1230-1233, 2006.

6. Abiko Y: Investigations on pantothenic acid and its related compounds. IX. Biochemical studies.4. Separation and substrate specificity of pantothenate kinase and phosphopantothenoylcysteine synthetase. J Biochem 61: 290-299, 1967.

7. Dansie LE, Reeves S, Miller K, Zano SP, Frank M, Pate C, Wang J and Jackowski S: Physiological roles of the pantothenate kinases. Biochem Soc Trans 42: 1033-1036, 2014.

8. Hörtnagel K, Prokisch $\mathrm{H}$ and Meitinger T: An isoform of hPANK2, deficient in pantothenate kinase-associated neurodegeneration, localizes to mitochondria. Hum Mol Genet 12: 321-327, 2003.

9. Brunetti D, Dusi S, Morbin M, Uggetti A, Moda F, D'Amato I, Giordano C, d'Amati G, Cozzi A, Levi S, et al: Pantothenate kinase-associated neurodegeneration: Altered mitochondria membrane potential and defective respiration in Pank2 knock-out mouse model. Hum Mol Genet 21: 5294-5305, 2012. 
10. Alfonso-Pecchio A, Garcia M, Leonardi R and Jackowski S: Compartmentalization of mammalian pantothenate kinases. PLoS One 7: e49509, 2012.

11. Kotzbauer PT, Truax AC, Trojanowski JQ and Lee VM: Altered neuronal mitochondrial coenzyme A synthesis in neurodegeneration with brain iron accumulation caused by abnormal processing, stability, and catalytic activity of mutant pantothenate kinase 2. J Neurosci 25: 689-698, 2005

12. Leonardi R, Zhang YM, Lykidis A, Rock CO and Jackowski S: Localization and regulation of mouse pantothenate kinase 2 . FEBS Lett 581: 4639-4644, 2007.

13. Leonardi R, Rock CO, Jackowski S and Zhang YM: Activation of human mitochondrial pantothenate kinase 2 by palmitoylcarnitine. Proc Natl Acad Sci USA 104: 1494-1499, 2007.

14. Levi S and Finazzi D: Neurodegeneration with brain iron accumulation: Update on pathogenic mechanisms. Front Pharmacol 5: 99, 2014.

15. Aoun M and Tiranti V: Mitochondria: A crossroads for lipid metabolism defect in neurodegeneration with brain iron accumulation diseases. Int J Biochem Cell Biol 63: 25-31, 2015.

16. Zizioli D, Tiso N, Guglielmi A, Saraceno C, Busolin G, Giuliani R, Khatri D, Monti E, Borsani G, Argenton F and Finazzi D KNock-down of pantothenate kinase 2 severely affects the development of the nervous and vascular system in zebrafish, providing new insights into PKAN disease. Neurobiol Dis 85: 35-48, 2016.

17. Rana A, Seinen E, Siudeja K, Muntendam R, Srinivasan B van der Want JJ, Hayflick S, Reijngoud DJ, Kayser O and Sibon OC: Pantethine rescues a Drosophila model for pantothenate kinase-associated neurodegeneration. Proc Natl Acad Sci USA 107: 6988-6993, 2010.

18. Srinivasan B, Baratashvili M, van der Zwaag M, Kanon B, Colombelli C, Lambrechts RA, Schaap O, Nollen EA, Podgoršek A, Kosec G, et al: Extracellular 4'-phosphopantetheine is a source for intracellular coenzyme A synthesis. Nat Chem Biol 11: 784-792, 2015.

19. Di Meo I, Colombelli C, Srinivasan B, de Villiers M, Hamada J, Jeong SY, Fox R, Woltjer RL, Tepper PG, Lahaye LL, et al Acetyl-4'-phosphopantetheine is stable in serum and prevents phenotypes induced by pantothenate kinase deficiency. Sci Rep 7: 11260, 2017.

20. Khatri D, Zizioli D, Tiso N, Facchinello N, Vezzoli S, Gianoncelli A, Memo M, Monti E, Borsani G and Finazzi D: Down-regulation of coasy, the gene associated with NBIA-VI, reduces Bmp signaling, perturbs dorso-ventral patterning and alters neuronal development in zebrafish. Sci Rep 6: 37660, 2016

21. Dusi S, Valletta L, Haack TB, Tsuchiya Y, Venco P, Pasqualato S, Goffrini P, Tigano M, Demchenko N, Wieland T, et al: Exome sequence reveals mutations in CoA synthase as a cause of neurodegeneration with brain iron accumulation. Am J Hum Genet 94 11-22, 2014.

22. Annesi G, Gagliardi M, Iannello G, Quattrone A, Iannello G and Quattrone A: Mutational analysis of COASY in an italian patient with NBIA. Parkinsonism Relat Disord 28: 150-151, 2016.
23. Grillo E, Ravelli C, Corsini M, Ballmer-Hofer K, Zammataro L, Oreste P, Zoppetti G, Tobia C, Ronca R, Presta M and Mitola S: Monomeric gremlin is a novel vascular endothelial growth factor receptor-2 antagonist. Oncotarget 7: 35353-35368, 2016.

24. Gatta LB, Vitali M, Verardi R, Arosio P and Finazzi D: Inhibition of heme synthesis alters amyloid precursor protein processing. J Neural Transm (Vienna) 116: 79-88, 2009.

25. Livak KJ and Schmittgen TD: Analysis of relative gene expression data using real-time quantitative pcr and the 2(-Delta Delta C(T)) method. Methods 25: 402-408, 2001.

26. Poli M, Derosas M, Luscieti S, Cavadini P, Campanella A, Verardi R, Finazzi D and Arosio P: Pantothenate kinase-2 (Pank2) silencing causes cell growth reduction, cell-specific ferroportin upregulation and iron deregulation. Neurobiol Dis 39: 204-210, 2010.

27. Siudeja K, Srinivasan B, Xu L, Rana A, de Jong J, Nollen EA, Jackowski S, Sanford L, Hayflick S and Sibon OC: Impaired Coenzyme A metabolism affects histone and tubulin acetylation in Drosophila and human cell models of pantothenate kinase associated neurodegeneration. Embo Mol Med 3: 755-766, 2011.

28. Campanella A, Privitera D, Guaraldo M, Rovelli E, Barzaghi C, Garavaglia B, Santambrogio P, Cozzi A and Levi S: Skin fibroblasts from pantothenate kinase-associated neurodegeneration patients show altered cellular oxidative status and have defective iron-handling properties. Hum Mol Genet 21: 4049-4059, 2012.

29. Vanetti C, Bifari F, Vicentini LM and Cattaneo MG: Fatty acids rather than hormones restore in vitro angiogenesis in human male and female endothelial cells cultured in charcoal-stripped serum. PLoS One 12: e0189528, 2017.

30. Schoors S, Bruning U, Missiaen R, Queiroz KC, Borgers G, Elia I, Zecchin A, Cantelmo AR, Christen S, Goveia J, et al: Fatty acid carbon is essential for dNTP synthesis in endothelial cells. Nature 520: 192-197, 2015.

31. Wong BW, Wang X, Zecchin A, Thienpont B, Cornelissen I, Kalucka J, García-Caballero M, Missiaen R, Huang H, Brüning $\mathrm{U}$, et al: The role of fatty acid $\beta$-oxidation in lymphangiogenesis. Nature 542: 49-54, 2017.

32. Brown WR and Thore CR: Review: Cerebral microvascular pathology in ageing and neurodegeneration. Neuropathol Appl Neurobiol 37: 56-74, 2011.

33. Carmeliet $\mathrm{P}$ and Ruiz de Almodovar C: VEGF ligands and receptors: Implications in neurodevelopment and neurodegeneration. Cell Mol Life Sci 70: 1763-1778, 2013.

34. Woltjer RL, Reese LC, Richardson BE, Tran H, Green S, Pham T, Chalupsky M, Gabriel I, Light T, Sanford L, et al: Pallidal neuronal apolipoprotein $\mathrm{E}$ in pantothenate kinase-associated neurodegeneration recapitulates ischemic injury to the globus pallidus. Mol Genet Metab 116: 289-297, 2015.

35. Wolfram-Gabel R and Maillot C: Vascular networks of the nucleus lentiformis. Surg Radiol Anat 16: 373-377, 1994. 